УДК 81

DOI: 10.33184/YVDK-2021-04-30.44

Р.Г. Гатауллин (о.ф.н., проф. БашГУ), Kaiser, Herbert (Prof. Dr.habil, Universität Bochum, Deutschland)

\title{
К ВОПРОСУ О СТРУКТУРЕ РЕДУПЛИКАЦИЙ В НЕМЕЦКОМ ЯЗЫКЕ
}

B статье исследуется периферийный способ словообразования - редупликачия. Анализируются простые, рифмованные способы, редупликация с абляутом. Указывается на стилистическую значимость редупликаџии в текстах СМИ и художественной литературы.

Ключевье слова: периферия деривачии, редупликация, простыле, рифмованные редупликации, стилистическая значимость редупликации

The article looks into the peripheral way of word formation reduplication. Simple, rhymed methods, reduplication with ablaut are analyzed. The stylistic significance of reduplication in media texts and fiction is pointed out

Key words: periphery of derivation, reduplication, simple, rhymed reduplications, stylistic significance of reduplication

Редупликация относится к периферийным видам словообразования современного немецкого языка. Левандовски определяет редупликацию как „vollständige oder teilweise Wiederholung der Wurzel, des Stammes oder des ganzen Wortes ohne oder mit teilweiser Abänderung des Lautbestandes; wortbildende sekundäre Verdoppelung von Wörtern oder primäre Doppelsetzung von Silben“ - как «полный или частичный повтор корня, основы или целого слова без или с частичным изменением звукового состава» [28, с.857; auch: 13, с. с.94-96].]. Значительная часть редупликации немецкого языка по своей структуре относится к простым (чистым, идентичным) повторам, ср.: ballaballa; dallidalli! dongdong; gaga ('etwas verrückt'); klickklick, klick(erdi)klick; penpeng; plemplem (sein); puffpuff; tamtam; töfftöff; Bonbon; Happihappi; междометия: ölohö; ölohö öloho; eilei, eileiei; Tamtam, 
hopphopp, dallidalli, balla-balla, Pinkepinke. Kindeskind, Helfershelfer (Selbstkomposita). Dallidalli, weitergehen! / Dalli, los, dalli, weitergehen; Pinkepinke, Kleinklein, killekille, hopphopp, dallidalli, balla-balla, plemplem, Mama, Papa, Pipi, Kuckuck, tamtam, dingding, dingdingding, tatata (rattern), soso, dodo, hamham, tocktock, hasemase; разговорные лексикализованные: tagtäglich, wortwörtlich, Kindeskind, Helfershelfer, Holzholz; grau-graue, graugraugraue Öde, tief-tiefe Sammetbläue, supersuperschnell, riesenriesengroß, riesenriesenriesengroß, Happihappi, Mampf(i)Mampf(i), Miammiam, Namnam/Njamnjam; междометия: Saufsauf, Sauf(i)-Sauf(i); наименование конкретного предмета: Рapa, Mama; Sing-Sing, Wauwau, Tamtam, Bimbim; Dringdring, Klingel-Klingel (Fahrradglocke), Klapp-Klapp, Gluckgluck (Getränk), Gluckerglucker (gluckern), Fress(i)-Fress(i), Fress-Fress (Essen), SchleckSchleck; Schluck-Schluck; производящие основы междометия gluck, глаголы (saufen, schlucken, fressen, glucken). Многие слова зафиксированы в словаре разговорного немецкого языка Хайнца Кюппера [27]: Bimbim: „Da kommt eine Bimbim“ Straßenbahn kindersprachl. Küpper 1987:108), Klapp-Klapp (hochklappbares Wandbett im Gefängnis [27, с.419]; в словарях Дудена plemplem (dumm, nicht recht bei Verstand: der ist ja plemplem) [11, c. 1156], все слова, относящиеся по своей структуре к композитам: Kindeskind, Helfershelfer.

В словаре Merriam-Webster редупликация определяется как "1: an act or instance of doubling or reiterating; 2a: an often grammatically functional repetition of a radical element or a part of it occurring usually at the beginning of a word and often accompanied by change of the radical vowel; $b(1)$ : a word or form produced by reduplication; (2): the repeated element in such a word or form; как 3. стилистическая фигура anadiplosis. Reduplication definition is - an act or instance of doubling or reiterating. How to use reduplication in a sentence. [17]. Дефиниция редупликации в английском языке подчеркивает ряд свойств, связанных с процессом словообразования, связанных с формой и значением: "Reduplication is a word-formation process in which meaning is expressed by repeating all or part of a word. As with morphology in general, two considerations that arise in reduplication are related to form and meaning. As for form, the term "reduplicant" has been widely used to refer to the repeated portion of a word, while "base" is 
used to refer to the portion of the word that provides the source material for repetition" [19, c.301-328; 23; 50; 52].

Простые редупликации (повторы без изменений) (Klimbim, Pinkepinke, Picknick, Tamtam): Effeff (etwas aus dem Effeff können, kennen, beherrschen), cp.: „Diese Mode-Hacks beherrschen modische Frauen aus dem Effeff! Der kaputte Reißverschluss, die zu engen Schuhe, der chaotische Kleiderschrank [39]; Film-Film, graugraue Hemden c функцией интенсификации семантического объема. „Graugraue Hemden sind unter allen grauen Hemden dadurch hervorgehoben, dass sie als besonders grau wahrgenommen werden" $[10$, c.91]. В разговорном языке и в речи детей происходит повтор слова, ср.: klein-klein, Klein-Klein (im Klein-Klein des Alltags), Wauwau, soso. „Wer den weiten Blick wagt, der verliert sich nicht im Kleinklein des Alltags, der kann mehr und tiefer sehen. Einher geht das mit einem offenen und tieferen Blick nach innen, einem Geredetsein und Verwurzeltsein" [24].

Plemplem [11, c.1158]: (salopp) unvernünftig, dumm: der ist ja plemplemp: Er ist total plemplem.(Er ist dumm) [5, c.61]. „Etwa, dass wir alle ein bisschen plemplem sind, wenn uns der Name eines Herrn nicht einfällt, den wir zuletzt als Latexwurst verkleidet auf dem Karnevalsball der Freiwilligen Feuerwehr Pinneberg gesehen haben?“ [51, c. 38]. „Der größte Teil der Menschheit war plemplem. [4, c. 131]. „Woher kommt Pinkepinke Moneten, Mammon, Zechinen, Marie, Groschen, Steine, Kies, Moos, Mäuse, Zaster, Zunder, Knete, Pinkepinke, Kröten, Pulver, Lappen, Eier, Kohle(n), Piepen. [53]. „Kohle, Cash und Pinkepinke [7]. Pinkepinke haben: viel Geld besitzen. Das lautmalerische, dem Klang der Münzen nachgebildete Wort kommt wohl aus griechisch pinax «Schüssel» Pinkepinke, auch: Pinke: Der aus der Gaunersprache stammende ugs. Ausdruck für «Geld» gehört zu einem nur noch landschaftlich gebräuchlichen Verb pinken "hämmern", hart auf etwas schlagen, sodass ein heller, metallischer Klang entsteht. [36]. Die Bimbim (Straßenbahn): „Mit der App "Bim Bim" bist Du immer bestens über die Abfahrten der Wiener Linien sowie der Linz AG Linien informiert. Die App zeigt Dir in einer übersichtlichen Anzeige die nächsten Abfahrten einer bestimmten Haltestelle an“. «Купить «Белка интерактивная мягконабивная "Bimbim", со звуковыми эффектами» [my-shop.ru > shop > product Bimbim Line stickers]. Klapp-Klapp: косметика «Klapp-Klapp»; Klappklapp-Cosmetics, Klap-Klapp - много музыки, 
слушай бесплатно; „Mit den Füsschen tapp tapp tapp, mit den Händchen klapp klapp klapp, einmal hin, einmal her, rund herum, es ist nicht schwer" [18]. Puff-Puff - название развлекательного центра в Екатеринбурге: «В прошлую пятницу посетил данное заведение. Честно говоря, думал, что за такую цену будет не очень, оказался не прав. Ребята работают от души». "Puff-puff is a sweet, party snack that is popular in Nigeria. It's one of those things I look forward to when I'm out with my mother back in Nigeria" [Gateway to Gastronomy: Nigerian Puf-Puff. // thegatewayonline.ca > 18.03.2020].

Относительно часто встречаются рифмованные повторы с изменением начальных звуков (Reimbildungen), ср.: kallepalle, mogelpogel, popelmopel, superduper, rikepike, silkepilke, andimandi, meikepeike, sebimebi, veramera, larsiparsi, udopudo, susipusi, Doppelmoppel, Kuschelmuschel, HaukePauke, AchimPachim, Happenpappen, Hausmaus, Hechtelmechtel (любовные аферы), Heckmeck, Hokuspokus, holter(di)polter, Klimbim, Klimperwimper (künstlich verlängerte Wimper), Kuddelmuddel (norddt. kudeln 'sudeln хаос, неразбериха), Larifari, Picknick, Remmidemmi, ruckzuck, rummelpummel, Schickmicki (сноб; спесивый, надменный, кичливый), MultiKulti, das Schorlemorle (Mischgetränk aus Wein und Saft - вино с газированной водой. Большой немецкорусский словарь.), Techtelmechtel и т.д.

Узуальное слово Schorlemorle, состоящее из лексической единицы die Schorle (напиток, состоящий из вина и сока или минеральной воды) [11, с.1149], часто употребляется в рецептах, ср.: „Ein einfaches und geniales DDR-Rezept für Schorlemorle mit Apfelsaft findest du hier! Viel Spaß beim zubereiten und genießen“" [40]. Krawall und Remmidemmi in der Stadthalle [2]. Иногда наблюдается игра слов, ср.: «Так вот, шорле - это летний напиток, как глювайн (глинтвейн) - зимний в Германии. И хочется бесконечно бродить по Саксонской Швецарии - Чешской Швейцарии, останавливаясь на перевалах в затерянных лесных кафешках, взять там запотевший холодный стаканчик живительного Anфельшорле. Anфельшорле (нем. Apfelschorle) это оригинальное немецкое название яблочного сока смешанного с минеральной водой с газом. Бывают вообще разные шорле, Schorlemorle - шорлеморле, как говорят немцы в шутку про белое (вино) с зельтерской (минералка из старинного источника 
Зельтерс-ан-дер-Лан). Вайншорле с красным вином [58]. В этимологических словарях о происхождении слова Schorlemorle имеются противоречивые факты. Словарь Дудена [12, с. 648] связывает с имеющим употребление ещё в 18 ом веке нижнебоварским словом. Schurlemurle. Клуге объясняет появление данного понятия через югозападное диалектное слово schuren (das mundartliche südwestdeutsche schuren (,sprudeln“) [25; 26, с. 651]. Заметим, что этимологический словарь Клуге указывает на употребление редкой фамилии Schurlemurle [26, с. 651]. Имеются и другие толкования значения и появления данного слова в языке: „Sommerzeit - Schorlezeit! Zu den beliebtesten Getränken in Rheinland-Pfalz gehört in der warmen Jahreszeit sowohl auf dem Balkon zu Hause als auch auf der Terrasse des gastronomischen Betriebs zweifellos die Schorlemorle - ein Mischgetränk aus Wein und Mineralwasser. Sicher ist, Schorle ist die Abkürzung von Schorlemorle. Was danach kommt, sind Spekulationen, von denen die französische Variante aber eindeutig die charmanteste ist. Danach kommt Schorlemorle vom Trinkspruch der Soldaten Napoleons, die sich gerne und oft mit "Toujours l'amour Immer wieder auf die Liebe!" zugeprostet haben. Es könnte ihnen auch "Schorlemorle" eingefallen sein" [54]. В связи с частым употреблением Schorlemorle в разговорной речи, уместно привести известную немцам историческую байку про Гете и студентов. «Как-то утомленный с дороги поэт зашел в кабачок и велел принести ему вина и воды, которые он выпил, смешав пополам. Сидевшие напротив студенты, скорее всего не ведая, кто перед ними, принялись подтрунивать над создателем «Фауста». Поэт на это произнес назидание в стихотворной форме, суть которого сводилась к следующему: «От одной лишь воды немеешь - как рыба в пруду. От вина глупеешь - как господа за столом. Не желая быть ни тем, ни другим, Гете предпочел пить вино пополам с водой». В немецкоязычном тексте упоминается название напитка Schorlemorle, ср.: Goethe, der Dichterfürst, reimte dereinst in Jena aus dem Stehgreif gegenüber Studenten, die ihn belächelten, weil er eine Schorlemorle trank:

Wasser alleine macht stumm, das zeigen im Wasser die Fische.

Wein alleine macht dumm, das zeigen die Studenten (варианm die Herrn) bei Tische. 
Und da ich keins von beiden will sein,

trinke ich Wasser vermischt mit Wein.

[Johann Wolfgang Goethe: Gedichte]

-Johann Wolfgang von Goethe - „In diesem Sinne - euch allen ein schönes Wochenende bei einem schönen Gläschen Weinschorle“.

Multikulti, MultiKulti: Когда попытки построить мультикультурное общество («мультикультурализм») в Германии, в котором представители различных культур должны были жить в полном согласии, окончательно и бесповоротно провалились, особо частотное употребление получило новое слово Multikulti, означающее ,ein friedliches Miteinander vieler Kulturen in deutschen Großstädten“" [30]. Wir fordern den Umzug des Multi-Kultis in die Inselstraße und damit die Pläne von Christos Chatziioannou $\mathrm{zu}$ genehmigen. [www.openpetition.de> petition > online > fuer-das-multik..]. "Multikulti is dead - or is it not? Shop window in Berlin-Neukoelln" [22]. Im Pariser Stadtteil Belleville leben viele Einwanderer. Leider ist das Zusammenleben nicht immer friedlich [www.dw.com > multikulti-in-..27.10.2020]. „Die deutsche multikulturelle Gesellschaft kann man sehr deutlich in Großstädten wie Berlin sehen. Hier gibt es eine Vielfalt an Kulturen, die alle zusammen in der Stadt leben. Multikulti wird hier täglich gelebt [34]. Multikulti auf dem Fußballfeld. Sie heißen Marin, Özil, Cacau oder Khedira. Sie sind die Hoffnung der deutschen Fußball-Fans bei der Weltmeisterschaft in Südafrika. Und: Sie haben ausländische Wurzeln [29].

Schickimicki связано со словом schick со значением «быть модно одетым»; Schickimicki - «пежон», «чувак»; «мелочь». "Die Männer-WCs, schwarz-weiß und mit viel modischem Schickimicki, wo man jedes Stäubchen sieht" [35]. Schickimicki: 1. Dandy, Geck; j-d, der sich betont modisch gibt; 2. modischer Kleinkram [11, c.1315]. „Es gibt viele Klischees über München, dass hier alles und alle schickimicki sind ist da nur ein weiteres. Wie bei allen Klischees liegt ein Körnchen Wahrheit. Schickimicki sind also auch junge Menschen in München und wir lieben die endlose Witzeschlange über den kleinen Justus-Salvator" [31]. Schickimicki Schnickschnack Musik trifft Handwerk. Handmade Schmuck mit Poesie die motiviert! Schickimicki Schnickschnack nennt sich das von Mirijam [32; 33]. Schickimicki выступает одновременно и в качестве существительного и прилагательного, что характерно для 
молодежного жаргона, сp.: Einen Kater von diesem schickimicki Blubberwasser. Du bist plötzlich so schickimicki (Вдруг ты стала такой важной). Hey, Schickimicki, bist du öfter hier? Ihr neuer Freund ist ein richtiger Schickimicki. Her new boyfriend is a real trendy type [38]. Частое употребление слов такого рода ведет к тому, что они становятся словами года, недели, определенной группь носителей языка. Так, слово „der Schickimicki“ стало словом недели, ср.: Wer von einem Schickimicki begrüßt wird, muss mit einem angedeuteten Wangenkuss und einer großen Parfumwolke rechnen - denn ,schick“" ist in diesem Fall noch untertrieben. [8]. Mit dem Schickimicki hat die Düsseldorfer Altstadt im Juni 2012 in Sachen Club Nachwuchs bekommen. Die 65 Quadratmeter große Location auf der Neustraße [37]. „Bei Knigge geht es nicht um Schickimicki, sondern darum, praktisch zu handeln", betont Reich. Und nennt ein Beispiel: So müsse nicht zwingend der Mann der Frau die Autotür aufhalten. Schließlich könne auch sie am Steuer sitzen und einem älteren Herrn auf dem Beifahrersitz beim Ein- und Aussteigen helfen [1].

Основные значения Schlampampen 1) schlampige, unordentliche Person [bayerische-schimpfwoerter.de]; 2) schlemmen [11, c.1325]. „Die Schlampampen - mit : Damenwahl extra Frauenkabarett mit Musik Wenn acht Frauen in knallbunten Schlauchkleidern vor ausverkauften Häusern über die Bühne fegen, auf Hitzewellen surfen und eine Selbsthilfegruppe für ungeliebte Speckrollen gründen, dann sind „Die Schlampampen“ am Werk! 20 Jahre Schlampampen - zum ihrem Jubiläum geben sie alles auf der Bühne! Ihr aktuelles Programm garnieren sie mit Lieblingsszenen und Kultfiguren aus zwei Jahrzehnten" [49].

Узуальное слово Techtelmechtel (фамильярно: любовная интрижка, шуры-муры): mit j-d ein Techtelmechtel haben (einen Flirt haben) [11, c. 1520], cp.: „es wurde gemunkelt, dass sie früher einmal ein Techtelmechtel mit einem amerikanischen Offizier gehabt habe. „Und kein Techtelmechtel mit dem Dienstmädchen“ - (He заглядывайся на прислугу). Употребление редупликации в качестве названия группы, ср.: Konzerte: hier spielt Techtelmechtel. Techtelmechtel spielt an Anlässen jeglicher Grösse. Von Grosskonzerten über Kleinkunstbühnen bis hin zu Firmenevents und privaten Veranstaltungen sind wir flexibel einsetzbar [46]; употребление в названии книги Levi-Ezequiel Zuckersüß: Unsere Welt liegt im Keller und schimmelt vor sich hin: ein Techtelmechtel 
mit der Chuzpe. Kein Techtelmechtel mit einer Nixe: Roman, Kindle Edition - 2019, - 79c. [55]; в названии вина: „2019er Techtelmechtel Cuvée trocken“; Слово Techtelmechtel привлекает внимание, поэтому в текстах СМИ занимает сильные позиции, ср.: «Jahrelang hatte Frankreichs Präsident eine "strategische Partnerschaft" mit Russland angestrebt. Die Nawalny-Affäre wirft seine Pläne über den Haufen» [3]; Techtelmechtel mit dem Feind [ff das Südtiroler Wochenmagazin 12.04.2018, https://www.ffbz.com/politik-wirtschaft/politik]. „Tom hat das ganze Jahr über geschuftet, um sich eine Karibik-Kreuzfahrt leisten zu können“ [47]. „Eine herrliche Nacht erlebte das Publikum bei der "Witwenresidenz", aufgeführt unter freiem Himmel auf dem Breitunger Burghügel" [48].

Неузуальное сложное слово (Kuschelmuschel) состоит из основ глагола (kuscheln) и существительного „,die Muschel“ (моллюска, раковина, ракушка, телефонная трубка, оболочка): „Wenn bei uns daheim ,Kuschelmuschel' angesagt ist, kommen immer ,Puschel' und ,Wuschel' ins Spiel. Das sind die kuschelweichen Teddys der Kleinen“ [16] „Da wollte ich jeden Tag auf der Terrasse frühstücken, in meiner Kuschelmuschel lesen oder schreiben“. "Auch, wenn das Wetter wirklich eher Kuschelmuschel-Stimmung macht". Название книги Даля Р. [6]. Sonneninsel / Kuschelmuschel für den Garten oder Ihren Wellnessbereich, aus wetterbeständigem Polyrattan anthrazitfarbenes Geflecht, hochwertig [41].

Слово superduper, имеющее значение super, exzellent, unglaublich, состоит из двух частей, причем вторая часть не обладает каким-либо значением и не имеет свободного функционирования; Таким образом, обладает характером уникального элемента. В русском языке имеет соответствие супепупер. $\quad B$ кембриджском словаре английского языка прилагательное (наречие) super-duper определяется как excellent. Примеры из словаря: “Тhey are both super-duper spellers". "He was one of these young guys who were just super-duper". "I've got a new super-duper computer". "They put the company's success down to their super-duper manager" [9]. Творческая страница Super Duper, на которой можно слушать и скачать музыку mp3 Super Duper онлайн бесплатно, смотреть видео [43; 44]. Nur wenige Einstellungen sind notwendig, damit Super Duper eine startfähige Kopie der Festplatte erstellt [45]. Der Clou: Auch beim Totalausfall der internen Festplatte könnt ihr euren Rechner einfach über das 
SuperDuper-Backup neu starten und seid so. „Du hast es echt gemütlich hier in diesem Haus und auf diesem superduper weichen Sofa. Er Kicherte" [Tara Tabea Gächter:Die Chronik der jungen Herrscherin: Die Befreiung - Band 1, BoD B Books on Demand 2020. - 264c. // books.google.ru > books]. Творческая страница Super Duper, на которой можно слушать и скачать музыку mp3 Super Duper онлайн бесплатно, смотреть видео, [44]. Книга сказок, автор Напп, Даниэль: Супер-пупер-поросенок [56] // https://www.labirint.ru/books/372142. «Пентагон разъяснил слова президента США Дональда Трампа о «супер-пупер» ракете. Об этом в четверг, 16 июля, сообщил телеканал CNN» [57].

В качестве общего вывода следует подчеркнуть, что редупликация несмотря на свою скромную роль в деривационном процессе, в некоторых видах текстов проявляет значительную активность в Интернет-текстах, в социальных сетях. Кроме того, редупликации являются средством варьирования художественного и публицистического выражения. В языках мира редупликации выражают различные грамматические и стилистические категории.

\section{ЛИТЕРАТУРА}

1. Beck, Nicolas: Bei Knigge geht es nicht um Schickimicki, ZeitJung - RNZ // www.rnz.de > zeitjung_18.09.2015

2. Bei Deichkind steht keiner lange still. www.vienna.at > krawall, 21.01.2019

3. Brändle, Stefan: Emmanuel Macron beendet das Techtelmechtel mit Moskau.// www.derstandard.de > story,16.09.2020

4. Bukowski, Charles: Ausgeträumt. Roman. Deutscher Taschenbuch Verlag, München 1997 (übersetzt von Carl Weissner). - 189 Seiten.

5. Bzdęga, Andrzej Zdzisław: Reduplizierte Wortbildung im Deutschen. Praca Wydana z Zasiłku Polskiej Akademii Nauk, Poznań 1965. - 129 Seiten

6. Dahl, Roald: Kuschelmuschel. Vier erotische Geschichten.: Vier erotische Überraschungen. Rowohlt Taschenbuch. - 2002. 192Seiten

7. Das Materialpaket rund ums Geld. // www.bildungsserver.de ', 4.03.2020 
8. Der Schickimicki Wort der Woche. DW 26.08.2013 // www.dw.com.der schickimicki

9. dictionary.cambridge.org > словарь > английский > su..].

10. Donalies, Elke: Die Wortbildung des Deutschen. Ein Überblick. Gunter Narr Verlag, Tübingen. 2002. - 190 Seiten.

11. Duden. Deutsches UniversalWärterbuch A-Z. Dudenverlag: Mannheim/ Wien/ Zürich 1989. - 1816c.

12. Duden, Bd. 7: Das Herkunftswörterbuch, 2. Aufl., Mannheim 1989. - 839c.

13. Fleischer, $W$., Barz, I. Wortbildung der deutschen Gegenwartssprache. De Gruyter, Tübingen 2012. - 484c. - c.9496.].

14. Gächter, Tara Tabea: Die Chronik der jungen Herrscherin: Die Befreiung - Band 1, BoD B Books on Demand 2020. - 264c. // books.google.ru > books

15. Gehört auf jeden Mac: SuperDuper und Mac Tracker // www.ifun.de > gehoert-auf-je. www.ifun.de > gehoert-aufje.26.06.2019

16. Guten Morgen Kuschelmuschel // www.rhein-zeitung.de

17. https://www.merriam-webster.com >

18. Humperdinck,E., Wette,A.: Hänsel and Gretel: A Fariry Opera in threeActs. // books.google.ru > books. 3.03.2020

19. Hurch, Bernhard, and Veronika Mattes. 2009. Typology of reduplication: The Graz database. // Use of databases in crosslinguistic studies. Edited by Martin Everaert, Simon Musgrave, and Alexis Dimitriadis, 301-328. New York: Mouton de Gruyter

20. Institut für Deutsche Sprache: Deutsches Referenzkorpus (DereKo-2017-II) / Archiv der Korpora geschriebener Gegenwartssprache. COSMAS II, C2API- Version 2.2.1 aktualisiert am 23. 06. 2017. Mannheim: Institut für Deutsche Sprache. (http://www.idsmannheim.de/DeReKo)

21. Goethe, Johann Wolfgang: Gedichte

22. Kantel, Gabriele: „MultiKulti is dead" and other ideas which are bad for the Balkans // www.esiweb.org > 17.10.2010

23. Kentner, Gerrit: Das Phänomen Reduplizierende Wortgebilde im Deutschen Osnabruck, 2011

24. Kleinklein des Alltags, // www.facebook.com > 3.02.2020

25. Kluge, Friedrich: Etymologisches Wörterbuch der deutschen Sprache, 22. Aufl., Berlin/New York 1989, c. 651] 
26. Kluge, Friedrich: Etymologisches Wörterbuch der deutschen Sprache. Walter de Gruyter 2015. - 887c.

27. Küpper, Heinz: Wörterbuch der deutschen Umgangssprache. PONS, 2009 (Digitale Bibliothek). - 959 Seiten.

28. Lewandowski, Th. Linguistisches Wörterbuch. Bd.2 Quelle\&Mezer; Heidelberg, Wiesbaden 1994. - 3 B-de 1287 S.

29. Multikulti auf dem Fußballfeld. www.dw.com> multikultiauf...22.06. 2010

30. MultiKulti: Neue Projekt- und Sprachassistentin aus Deutschland // multikulti.org.ua) neue-proje; www.alumniportaldeutschland.org >.

31. München, wie schickmicki seid ihr wirklich? mitvergnuegen.com > muench

32. Musik trifft Handwerk - schicki-schnacks Webseite. www.schickimicki-schnickschnack.de

33. Musik trifft Handwerk - schickmicki-schnickschnacks Webseite. // www.schickimicki-schnickschnack.de. 11.03.2018

34. Nissen, Shan Mari: Multikulti Berlin // portals.clio.me , deutschland > interkulturelle-identitaet 14.01.2020

35. Rundum sehr schön renoviert //www.suedostschweiz.ch, 07.12.2008

36. Schemann, Hans: Deutsche Idiomatik. Wörterbuch der deutschen Redewendungen im Kontext. De Gruyter 2011. - 1040c. https://doi.org/10.10.1515

37. Schickimicki - Düsseldorfll www.duesseldorf-tonight.de `. 28.06.2012

38. Schickimicki: Немецко-английский словарь. //dictionary.cambridge.org > немецко-английский словарь .28.10.2020

39. Schmidt, P.: Nerven“.// www.pinterest.ch > pin. Дата обращения 20.10.2020

40. Schorlemorle mit Apfelsaft. // ddr-rezepte.net ' Getränke. $13.01,2019$

41. Sonneninsel Kuschelmuschel - Das Relaxmöbel aus Polyrattan Jetzt als Komplettpacket! // sites.google.com > hgtyrrryrt

42. Store, App: Bim-Bim - Apple // apps.apple.com > app > bim-bim, 31.03.2020

43. Super-Duper -музыка, видео// www.realrocks.ru > Музыканты.20.04.17 > 
44. Super-Duper-музыка, видео// www.realrocks.ru >14.07.2019 >

45. SuperDuper: Startfähigen Festplatten-Klon erstellt. // www.macwelt.de > news > Wo.9.06.2020

46. Techtelmechtel - osteuropäische Weltmusik. // techtel-mechtel.ch

47. Techtelmechtel auf der Kreuzfahrt par Doreen Dose.//. www.cultura.com > techtelmec

48. Techtelmechtel im Mückenschwarm in Thüringen. // www.insuedthueringen.de >, 21.06.2011

49. Tickets für Die Schlampampen bei ProTicket kaufen. // www.proticket.de > ... > Show

50. Urbanczyk, Suzanne: Reduplication. 2017 // Reduplication Linguistics - $\quad$ Oxford Bibliographies https://www.oxfordbibliographies.com >;

51. Weiler, Jan: Mein Leben als Mensch. Illustriert von Larissa Bertonasco. 2. Auflage. Rowohlt, Reinbek 2009. - 229 Seiten.

52. Wiese, R.: Über die Interaktion von Morphologie und Phonologie Reduplikation im Deutschen. ZPSK 43.1990. - 603-624

53. Woher kommt Pinkepinke // www.wissen.de〉 wortherkunft. 05.04.2019

54. Zimmermann, Frieder: Toujours l'amour - Immer wieder auf die Liebe II https://www.agrarpresseportal.de/nachrichten/toujours 06.08.2010

55. Zuckersü $\beta$, Levi-Ezequiel: Unsere Welt liegt im Keller und schimmelt vor sich hin: ein Techtelmechtel mit der Chuzpe. kindle edition, 2019. - 138c.; Rowenta Crane: Kein Techtelmechtel mit einer Nixe: Roman, Kindle Edition - 2019, 79c.;

56. Hann, Даниэль: Супер-пупер-поросенок https://www.labirint.ru/books/ 372142.

57. Пентагон рассказал об испытаниях «супер-пупер» ракеты Трампа Известия iz.ru > Новость > Мир, 17.07.2020

58. Шорле - прохлада в лето: u kuzi - LiveJournal- // ukuzi.livejournal.com >. 24.08.2011

(C) Гатауллин Р.Г., Keiser Herbert 2021 г 